\title{
Tenacidade à Fratura Translaminar Dinâmica de um Laminado Híbrido Metal-Fibra para Uso em Elevadas Temperaturas
}

\author{
José R. Tarpani, Maria C. A. Gatti \\ Departamento de Engenharia de Materiais, Aeronáutica e Automobilística, EESC-USP
}

\begin{abstract}
Resumo: A tenacidade à fratura translaminar dinâmica do laminado híbrido metal-fibra titânio-grafite com matriz termoplástica foi determinada sob as velocidades de impacto de 2,25 e $5,52 \mathrm{~m} / \mathrm{s}$, no intervalo de temperaturas de -196 a $+180{ }^{\circ} \mathrm{C}$, e comparada à de laminados compósitos convencionais de fibras de carbono e resina epóxi. Constatou-se que o laminado híbrido exibe uma tenacidade à iniciação da fratura inferior à dos compósitos tradicionais com fibras na forma de fita unidirecional, porém superior à dos laminados convencionais com fibras na forma de tecido bidirecional. Os ensaios de impacto revelaram que, comparativamente ao desempenho mecânico dos laminados carbono-epóxi, o emprego do laminado híbrido metal-fibra se justifica mais pela sua resistência à propagação do que à iniciação da fratura dinâmica.
\end{abstract}

Palavras-chave: Impacto instrumentado Charpy, laminado híbrido metal-fibra, tenacidade à fratura dinâmica.

\section{Translaminar Dynamic Fracture Toughness of a Hybrid Fiber-Metal Laminate Devised to High-Temperature Applications}

Abstract: The translaminar dynamic fracture toughness of titanium-graphite hybrid fiber-metal laminate with thermoplastic matrix has been determined at the impact velocities of 2.25 and $5.52 \mathrm{~m} / \mathrm{s}$, within the temperature range from -196 to $+180{ }^{\circ} \mathrm{C}$, and compared to that of conventional carbon-epoxy composite laminates. The hybrid laminate exhibits lower initiation fracture toughness than traditional unidirectional tape composites though it is tougher than conventional woven fabric laminates. Impact tests revealed that, if compared to the mechanical performance of conventional carbon-epoxy laminates, the fiber-metal laminate application must rely on its resistance to dynamic fracture propagation rather than on fracture initiation.

Keywords: Dynamic fracture toughness, hybrid fiber-metal laminate, instrumented Charpy impact testing.

\section{Introdução}

Os tradicionais laminados compósitos de fibras de carbono e resina epóxi, denominados de ora em diante simplesmente como C-EPX, em que fibras contínuas de carbono reforçam uma matriz epoxídica, satisfazem os atuais requisitos de projetos estruturais de aeronaves subsônicas. Entretanto, aeronaves supersônicas requerem materiais cujas propriedades mecânicas sejam mantidas em elevadas temperaturas de serviço. A Companhia Aeroespacial Boeing é uma das responsáveis pelo projeto americano HSCT (High Speed Civil Transport), e desenvolveu juntamente à Agência Aeroespacial Americana - NASA o laminado híbrido metal-fibra (LMF) TiGra, formado por lâminas alternadas de liga de titânio (Ti) e de compósito de matriz polimérica termoplástica PEEK (poli-éter-éter-cetona) reforçado com fibras de grafite (Gra), de modo a satisfazer os critérios mais severos do projeto HSCT em que temperaturas de até $180^{\circ} \mathrm{C}$ são previstas para fuselagens de aeronaves se deslocando a velocidades de Mach 2, $5^{[1]}$.

Uma limitação amplamente reconhecida das estruturas laminares é a sua baixa resistência a impactos transversais ${ }^{[2]}$. Entretanto, o desempenho dos laminados compósitos aeronáuticos sob trincamento translaminar dinâmico não foi até o momento devidamente apreciado, particularmente no caso dos LMF.

A partir dos conceitos da Mecânica da Fratura, é possível inferir o grau de segurança que um componente estrutural possui contra fraturas catastróficas em serviço. Um dos parâmetros da Mecânica da Fratura Elásto-Plástica (MFEP) empregado na estimativa da resistência dos materiais à fratura lenta (quase-estática), sob condições de plasticidade à frente da trinca, é a integral- $\mathrm{J}^{[3]}$, que originou o critério de tenacidade à iniciação da fratura $J_{i c}$. Uma vez determinado experimentalmente, $J_{i c}$ deve ser validado de modo a garantir que a zona plástica na ponta da trinca, englobando a zona de processos de fratura, seja pequena quando comparada às dimensões do componente. Caso esta condição seja satisfeita, assume-se que a tenacidade $J_{i c}$ é uma verdadeira propriedade do material, independentemente do tamanho e da geometria do componente, sendo então designado $J_{I C}$ e garantindo uma condição de deformação predominantemente plana no evento da fratura.

No presente trabalho, os conceitos da MFEP tradicional, por intermédio da integral-J, é estendido ao regime de carregamento dinâmico sob impacto Charpy de um laminado híbrido metal-fibra do tipo TiGra possuindo defeitos translaminares, dando origem ao critério de início da fratura rápida $J_{i d}$.

É realizada uma comparação, numa base de propriedade mecânica absoluta (i.e., por unidade de volume do corpo de prova), da tenacidade à fratura $J_{i d}$ do LMF-TiGra e de laminados convencionais C-EPX ${ }^{[4]}$, sob duas taxas de carregamento e num amplo intervalo de temperaturas.

Não obstante as velocidades de impacto aplicadas no presente estudo sejam relativamente baixas, até $5,5 \mathrm{~m} / \mathrm{s}(20 \mathrm{~km} / \mathrm{h})$, a sua aplicabilidade num contexto da engenharia aeronáutica se justifica em virtude da relativamente frequente ocorrência de eventos envolvendo choques entre veículos circulando pela pista e aeronaves estacionadas, entre aeronaves taxiando em solo, assim como choques da cauda de aeronaves contra o solo, seja durante sua a decolagem ou aterrissagem. Desta forma, os resultados apresentados neste trabalho em termos de tenacidade à fratura dinâmica podem ser potencialmente utilizados na qualificação e/ou seleção de materiais de construção aeronáutica sob condições factíveis de serviçoco ${ }^{[5,6]}$. 


\section{Materiais e Corpos de Prova}

\section{Laminado híbrido metal-fibra TiGra}

Placas do laminado híbrido TiGra foram confeccionadas na Universidade de Liverpool-UK, a partir do empilhamento alternado de 3 lâminas de Ti comercialmente puro e 2 laminados com 7 camadas de pré-impregnado (APC-2 da ICI-Fiberite ${ }^{\circledR}$ ) de matriz termoplástica PEEK (poli-éter-éter-cetona) reforçada com $60 \%$ em volume de fibras unidirecionais (arranjo fita) de grafite (AS4 da Hercules $\left.^{\circledR}\right)^{[7,8]}$.

Um filme de PEEK puro com 0,15 mm de espessura foi colocado em cada interface metal/compósito, sendo que as faces das lâminas metálicas foram jateadas com areia de modo a eliminar possíveis camadas de óxido e impurezas, bem como introduzir rugosidade superficial para favorecer o ancoramento mecânico da fase polimérica. Através de medidas realizadas numa superfície metálica delaminada do TiGra, obteve-se um índice de rugosidade superficial média $\left(\mathrm{R}_{\mathrm{a}}\right)$ da ordem de $2,4 \mu \mathrm{m}$, que pode ter sido subestimado em decorrência de eventuais resíduos de polímero PEEK na superfície do metal.

A consolidação do produto final segundo um arranjo $3 / 2\left(0^{\circ}\right)_{7}$ foi realizada por compressão a cerca de $400{ }^{\circ} \mathrm{C}$ em molde fechado, seguida por resfriamento controlado e alívio de tensões residuais. O LMF-TiGra, com espessura final de $5 \mathrm{~mm}$ e exibiu as seguintes porcentagens em volume de seus componentes individuais: $20 \%$ de polímero PEEK, $25 \%$ de fibras de grafite, e $55 \%$ de Ti, conforme estimativa realizada por análise computadorizada de imagens de seções transversais e longitudinais de amostras materialograficamente polidas.

A Figura 1 mostra uma vista em corte da seção longitudinal do LMF-TiGra, assim como uma ampliação da região de interface entre as fases metálica e do compósito do laminado híbrido TiGra. Observa-se nesta vista ampliada a extensiva rugosidade criada na superfície metálica por jateamento de areia durante o processo de preparo das lâminas de Titânio visando favorecer a interação física do polímero nos vários pontos gerados de ancoramento mecânico. É possível ainda notar a presença de uma região rica em PEEK, originada pelo filme de polímero termoplástico intencionalmente alocado naquela posição, bem como confirmar a disposição exclusivamente unidirecional das fibras de reforço de grafite, que se mostram adequadamente distribuídas na matriz polimérica termoplástica.

Por intermédio de análise dinâmico-mecânica e calorimetria exploratória diferencial, a temperatura de transição vítrea $\left(\mathrm{T}_{\mathrm{g}}\right)$ do compósito PEEK/Gra, que corresponde a uma fração volumétrica de $45 \%$ no laminado híbrido TiGra, foi determinada entre 160 a $185{ }^{\circ} \mathrm{C}^{[9]}$. O módulo de armazenamento (E') do compósito foi estimado em 44,2 $\mathrm{GPa}$, enquanto que seu módulo de perda (E") alcançou o valor de 67,2 MPa. Neste mesmo trabalho, foi demonstrado que a rota de fabricação empregada para a produção do laminado TiGra, com a solidificação do polímero se dando através de resfriamento controlado, produziu um compósito PEEK/ Gra com propriedades termo-mecânicas otimizadas com relação a outros possíveis ciclos térmicos, tais como por resfriamento lento (recozimento) ou rápido (têmperas em água e nitrogênio líquido). A análise por calorimetria exploratória aferiu uma temperatura de fusão cristalina $\left(\mathrm{T}_{\mathrm{f}}\right)$ de $343^{\circ} \mathrm{C}$, precedida por um pico de cristalização $\left(\mathrm{T}_{\mathrm{c}}\right)$ ocorrendo a $173^{\circ} \mathrm{C}$, o qual indicou um teor mássico de cristalinidade do polímero PEEK de $33 \%$.

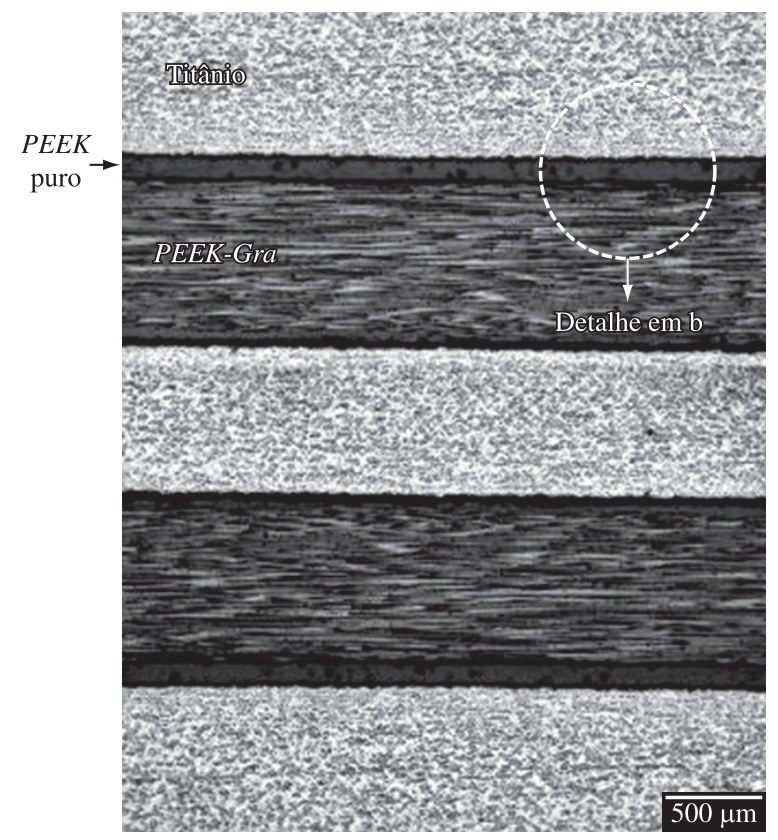

(a)

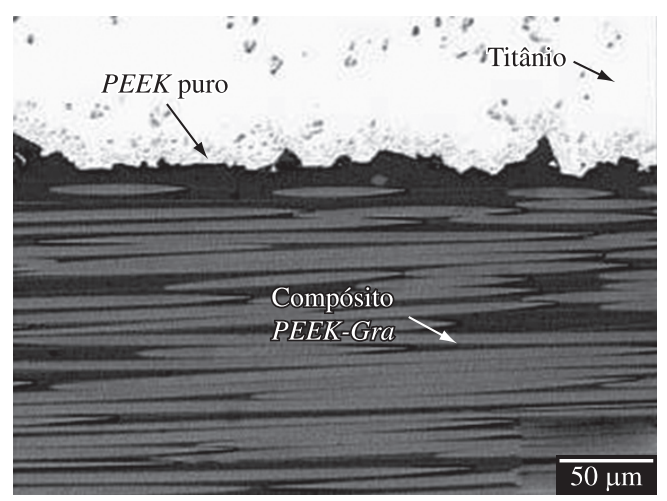

(b)

Figura 1. a) Corte da seção longitudinal do LMF-TiGra; e b) vista detalhada das fases e interfaces presentes no laminado híbrido metal-fibra.

\section{Corpos de prova (Cdps)}

Os cdps foram extraídos por corte com jato d'água das placas originais de TiGra, na forma de barras de flexão em três pontos nas dimensões nominais de $(5 \times 10 \times 55) \mathrm{mm}^{3}$.

O entalhamento dos cdps foi realizado com um disco diamantado com 0,5 mm de espessura sob baixa rotação e refrigeração com água, seguindo procedimento adotado em diversos trabalhos publicado na literatura científica sobre fratura translaminar em laminados compósitos convencionais e híbridos metal-fibra submetidos a carregamentos quase-estáticos, cíclicos e dinâmicos ${ }^{[10-14]}$.

$\mathrm{O}$ posicionamento do entalhe foi de tal modo a provocar a fratura translaminar dos laminados, exatamente como realizado em recente trabalho pelos autores ${ }^{[4]}$.

Considerando que as fibras de grafite e as lâminas de Ti foram dispostas em uma única orientação (longitudinal-L, correspondente à direção principal $0^{\circ}$ ), o entalhe Charpy foi posicionado perpendicularmente àquela direção de máxima resistência mecânica do LMF. Assim sendo, e seguindo-se a nomenclatura originalmente estabelecida pela ASTM-E399 ${ }^{[15]}$, os cdps assumiram uma orientação L(ongitudinal)-T(ransversal). 


\section{Procedimentos}

\section{Ensaio de impacto Charpy instrumentado}

Os ensaios de impacto Charpy instrumentado foram realizados em um sistema Instron-Wolpert ${ }^{\circledR} \mathrm{PW} 30$ com fundo de escala máximo de $300 \mathrm{~J}$, integrado a um microcomputador que opera programas computacionais destinados à leitura e ao tratamento dos dados para interpretação dos resultados finais.

Maiores detalhes sobre o sistema Charpy Instron-Wolpert ${ }^{\circledR}$ PW30 e acerca dos procedimentos de ensaio de tenacidade à fratura translaminar de laminados compósitos aeronáuticos podem ser obtidos no trabalho de Tarpani \& Gatti ${ }^{[4]}$.

Cdps do LMF-TiGra foram impactados nas temperaturas de, respectivamente, $-196,-70,23$ e $180{ }^{\circ} \mathrm{C}$, e sob duas diferentes velocidades de impacto do martelo, respectivamente de 2,25 e $5,52 \mathrm{~m} / \mathrm{s}$.

\section{Ponto de iniciação do trincamento sob carregamento dinâmico}

Para a determinação do ponto, em um diagrama de carga aplicada $(P)$ contra a deflexão na linha de carregamento $(s)$ do cdp Charpy, em que realmente se inicia o trincamento do espécime previamente entalhado e submetido a um impacto translaminar, empregou-se a metodologia analítica desenvolvida por Kobayashi et al. ${ }^{[16]}$, denominada Método da Taxa de Variação da Flexibilidade Elástica (MTVFE). Ao leitor é sugerida a leitura de Tarpani \& Gatti ${ }^{[4]}$ de modo a obter informações mais detalhadas a respeito do método.

\section{Cálculo da tenacidade à fratura dinâmica na iniciação do trincamento, $\mathrm{J}_{\text {id }}$}

$\mathrm{Na}$ determinação da tenacidade à fratura sob carregamento dinâmico, $J_{i d}$, empregou-se a Equação 1, desenvolvida para trincas

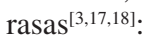

$$
J_{i d}=1,46 \frac{E_{i}}{B \cdot b}
$$

onde $E_{i}$ é a energia absorvida pelo cdp até o início de seu trincamento (definida essencialmente como a área sob o diagrama $P-s$, até aquele ponto de iniciação definido no item anterior), e $B$ e $b$ são, respectivamente, a espessura e o ligamento original do cdp, ou seja, eles compõem a seção transversal retangular íntegra do corpo de prova, à frente do entalhe.

\section{Cálculo da tenacidade à fratura dinâmica na carga máxima, $J_{m d}$}

Uma maneira mais simples e direta de se estimar a tenacidade à fratura dinâmica dos materiais admite que o defeito (trinca ou entalhe) inicia seu crescimento exatamente no ponto de carga máxima suportada pelo cdp ${ }^{[17]}$. Esta definição originou o critério $J_{m d}$, determinado a partir da Equação 1, porém utilizando-se, desta feita, a energia consumida pelo espécime até a carga máxima $\left(E_{m}\right)$.

\section{Validação dos valores de tenacidade}

Para se determinar a validade, ou não, dos resultados de tenacidade à fratura elasto-plástica, de forma a que esta quantidade possa, ou não, ser considerada uma propriedade intrínseca e verdadeira do material, empregam-se os chamados critérios de validade (no presente caso, fornecidos na unidade de milímetros) já bem estabelecidos na literatura $^{[19]}$, tendo-se como base as dimensões do cdp. De modo resumido, são estes os critérios de validação de $J_{i d}$ e $J_{m d}$ (Equação 2):

$$
B, b, W, a>25\left(\frac{J_{i d(m d)}}{\sigma_{e d}}\right)
$$

onde $W$ é a largura do cdp, $a$ o comprimento do entalhe, e $\sigma_{e d}$ é, no caso dos materiais metálicos, o limite de escoamento dinâmico do material ${ }^{[20]}$ e $P_{e d}$ a carga de escoamento correspondente (Equação 3), onde:

$$
\sigma_{e d}=\frac{2,85 P_{e d} W}{\left(B . b^{2}\right)}
$$

Neste trabalho, o parâmetro $\sigma_{e d}$ foi determinado com base em uma saída da linearidade inicial da curva polida de carga $(P)$ contra deflexão do cdp $(s)$, da ordem de 5\%. A Figura 2 plota a curva $P-s$ do TiGra ensaiado à temperatura de $-70{ }^{\circ} \mathrm{C}$ e sob uma velocidade de impacto de $2,25 \mathrm{~m} / \mathrm{s}$. Não obstante nesta temperatura o polímero PEEK esteja operando muito abaixo de sua transição vítrea $\left(\mathrm{T}_{\mathrm{g}}\right)$, que é da ordem de $150{ }^{\circ} \mathrm{C}$, a não-linearidade exibida pela respectiva curva $P-s$ denota efeitos de plasticidade devida à presença do polímero termoplástico bem como da fase metálica intrinsecamente dúctil.

Um programa computacional contemplando o MTVFE para determinação do ponto de início de trincamento, assim como as formulações providas nas Equações 1-3, foi elaborado em ambiente Matlab $^{\circledR}$, possibilitando o polimento das curvas $P-s$ originais e a imediata geração e validação (ou não) dos resultados de tenacidade segundo as duas abordagens-J em questão, a saber, de iniciação de trincamento, $J_{i d}$, e de carga máxima, $J_{m d}$.

\section{Análise estatística}

A análise estatística realizada neste trabalho teve como referência o método $t$-Student, visto que o número de amostragem foi pequeno $(\mathrm{n}<<30)^{[21]}$.

O limite de confiabilidade empregado no estudo foi de $95 \%$, e o de significância de 5\%, com 4 graus de liberdade, o que corresponde a um valor tabelado $t$-Student de 2,13 ${ }^{[22,23]}$.

As análises realizadas foram bilaterais, empregando-se como dados de entrada os valores de tenacidade $\mathrm{J}$ e o critério de validade, os quais foram calculados de forma convencional em termos de valores médios e correspondentes desvios-padrão, como funções, respectivamente, da temperatura e da taxa de carregamento aplicada no ensaio de impacto Charpy.

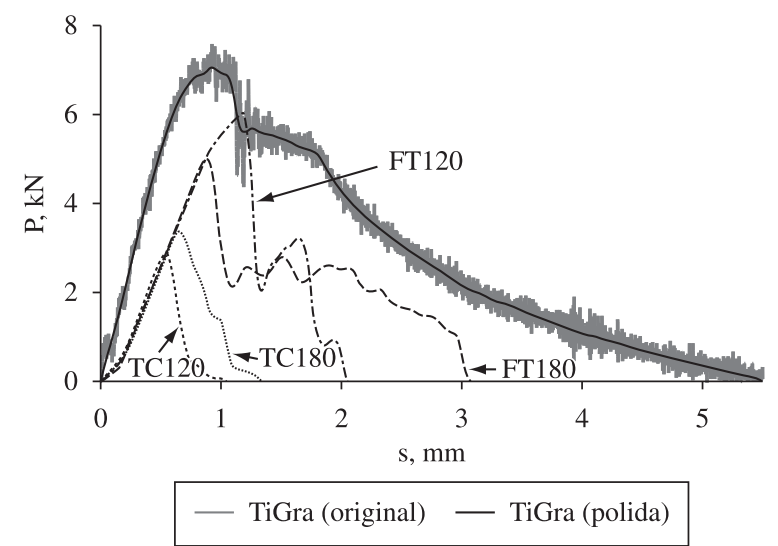

Figura 2. Curvas de carregamento original (contendo as oscilações naturais do processo de impacto) e polida segundo o Método da Média Móvel em rotina Matlab ${ }^{\circledR}$ para o laminado híbrido TiGra carregado a uma velocidade de $2,25 \mathrm{~m} / \mathrm{s}$ sob uma temperatura de $-70{ }^{\circ} \mathrm{C}$. As curvas de carregamento de quatro diferentes laminados convencionais $\mathrm{C}-\mathrm{EPX}^{[4]}$, ensaiados em idênticas condições que o TiGra, são traçadas para fins de comparação. A nomenclatura adotada para os compósitos C-EPX é: FT120 = Fita processada a $120{ }^{\circ} \mathrm{C}$, FT180 $=$ Fita processada a $180{ }^{\circ} \mathrm{C}$, TC120 $=$ Tecido processado a $120{ }^{\circ} \mathrm{C}$, $\mathrm{TC} 180=$ Tecido processado a $180{ }^{\circ} \mathrm{C}$. 
A análise estatística foi realizada em comparação com duas médias para cada critério de tenacidade J, e sua respectiva validação com base nas dimensões dos corpos de prova. Numa primeira abordagem, utilizou-se como parâmetro a variação da temperatura em cada ensaio realizado sob uma das duas taxas de carregamento empregadas no estudo. Na segunda abordagem, adotou-se como parâmetro a variação da velocidade de impacto para cada uma das quatro temperaturas em que o laminado TiGra foi avaliado.

\section{Resultados e Discussão}

A Figura 3 apresenta os resultados de ensaios de tenacidade $J_{i d}$ e $J_{m d}$ do TiGra considerando-se as várias temperaturas e taxas de carregamento empregadas no estudo. São plotados os valores médios dos parâmetros J obtidos a partir de três ensaios para cada condição experimental, com os respectivos desvios-padrão fornecidos na forma de barras verticais.

A união dos pontos de dados por intermédio de linhas tracejadas visa apenas indicar a tendência de comportamento dos resultados experimentais obtidos, não devendo, em princípio, ser empregada para fins de interpolação nem de extrapolação de dados.

Como linha de base são utilizados os resultados obtidos para quatro laminados convencionais C-EPX ${ }^{[4]}$.

A análise destes gráficos permite as seguintes observações, as quais são divididas em quatro seções específicas de maneira a facilitar a tarefa do leitor.

\section{Comportamento geral}

O laminado TiGra exibe valores de tenacidade à fratura $J_{i d}$ muito inferiores aos dos laminados C-EPX fita (FT), porém significativamente superiores aos dos C-EPX tecido (TC). Conforme observado em trabalho anterior dos autore ${ }^{[4]}$, uma das principais razões é o número de interfaces passíveis de delaminação, sendo 27 para os FT, contra 18 interfaces no TiGra, e apenas 13 dos TC, já que a delaminação constitui um poderoso mecanismo de tenacificação nas estruturas laminadas.

A Figura 4 comprova a ocorrência de delaminações, dentre outros mecanismos de fratura e tenacificação, num espécime de TiGra impactado sob baixa temperatura.

Os valores $J_{m d}$ do laminado TiGra são bastante próximo do laminado C-EPX FT120, e notavelmente superiores aos dos C-EPX TC. A rigor, o parâmetro $J_{m d}$ já computa, no caso de materiais mais tenazes, uma boa parcela de energia associada ao estágio de propagação da fratura no material, e os resultados obtidos indicam que os laminados TiGra e C-EPX FT já incorporam uma certa parcela de crescimento de danos anteriormente à carga máxima. Esta condição pode ser matematicamente expressa por uma razão $J_{m d} / J_{i d} \gg>1$, conforme pode ser observado nas Figuras 5a (para o TiGra) e 5b (especialmente para os laminados C-EPX FT). A fragilidade intrínseca dos laminados C-EPX TC, em especial do curado a $120^{\circ} \mathrm{C}$ ( $\left.\mathrm{TC} 120\right)$ é matematicamente representada por uma razão $J_{m d} / J_{i d} \approx 1$, denotando o início do trincamento ocorrendo exatamente na carga máxima suportada pelo corpo de prova.

Para o TiGra, além das delaminações, as quais se desenvolvem especialmente nas interfaces metal/polímero (Figura 4) em virtude da tipicamente baixa adesão aos substratos proporcionada pelos termoplásticos apolares, contribuem para a sua tenacidade à fratura a reconhecida ductilidade das fases polimérica termoplástica e

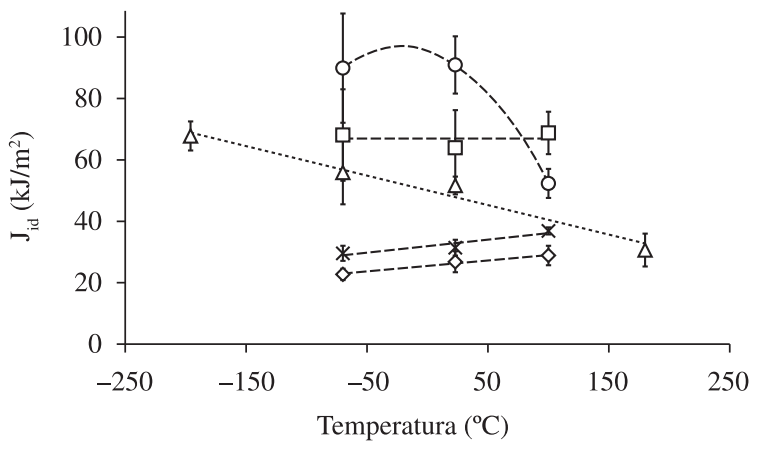

$\Delta$ TiGra $2,25 \mathrm{~m} / \mathrm{s} \circ \mathrm{FT} 120 \square \mathrm{FT} 180 \diamond \mathrm{TC} 120 \times \mathrm{TC} 180$

(a)

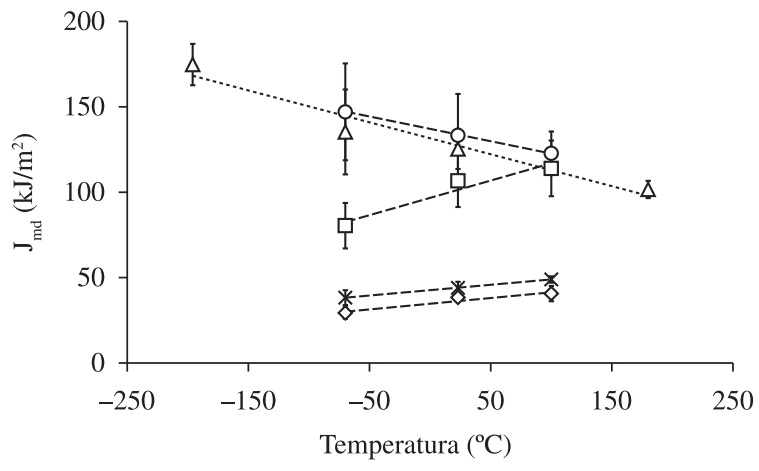

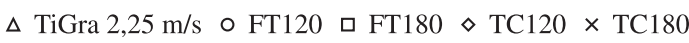

(c)

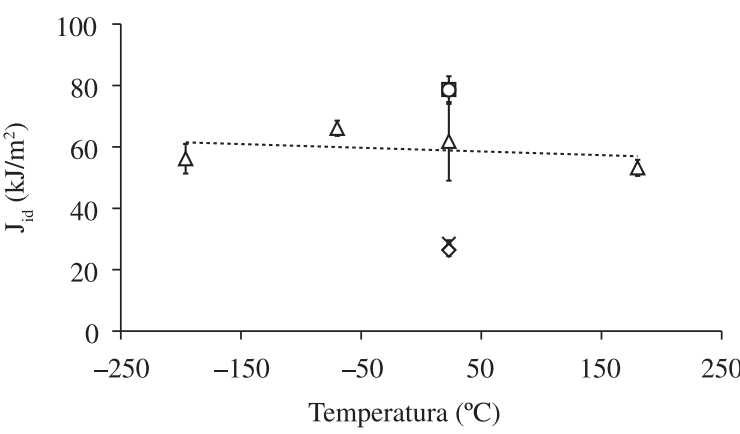

$\Delta$ TiGra $5,52 \mathrm{~m} / \mathrm{s} \circ \mathrm{FT} 120 \square \mathrm{FT} 180 \diamond \mathrm{TC} 120 \times \mathrm{TC} 180$

(b)

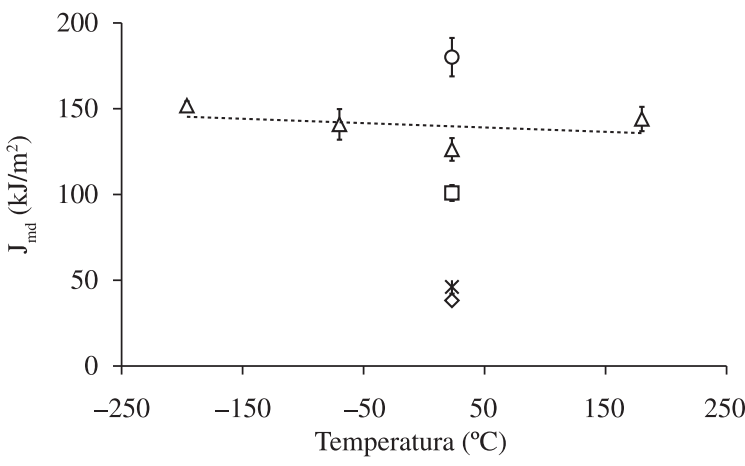

$\Delta$ TiGra $5,52 \mathrm{~m} / \mathrm{s} \circ \mathrm{FT} 120 \square \mathrm{FT} 180 \diamond \mathrm{TC} 120 \times \mathrm{TC} 180$

(d)

Figura 3. Resultados de tenacidade $J_{i d}$ e $J_{m d}$, para as diversas temperaturas de ensaio e velocidades de impacto: a) $J_{i d}$ a $2,25 \mathrm{~m} / \mathrm{s}$; b) $J_{i d}$ a $5,52 \mathrm{~m} / \mathrm{s}$; c) $J_{m d}$ a $2,25 \mathrm{~m} / \mathrm{s}$; e d) $J_{m d}$ a $5,52 \mathrm{~m} / \mathrm{s}$. 

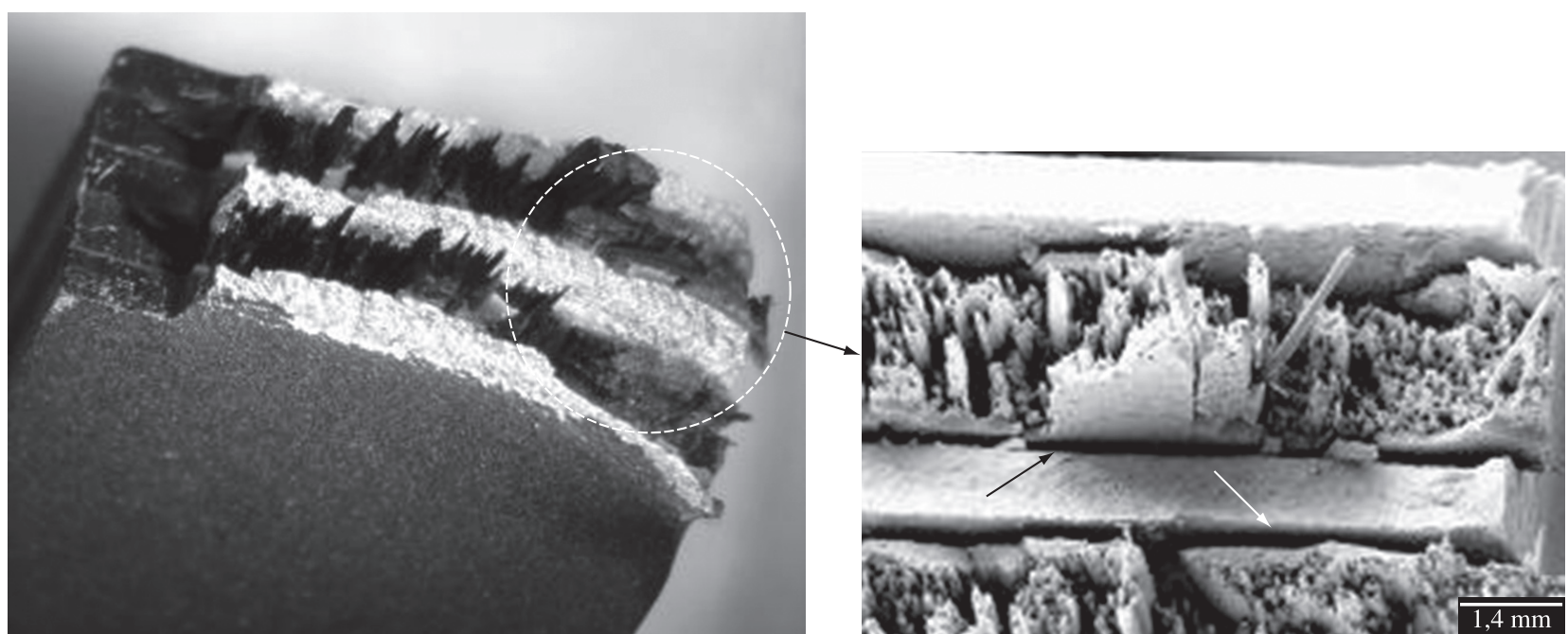

Figura 4. Aspecto de fratura de um espécime TiGra ensaiado a $2,25 \mathrm{~m} / \mathrm{s} \mathrm{e}-70{ }^{\circ} \mathrm{C}$. As delaminações são indicadas por setas. Note a ocorrência de quebra e arrancamento de fibras de reforço de grafite, além da deformação e trincamento das matrizes metálica (Titânio) e polimérica (PEEK).

metálica que compõem o laminado híbrido, enquanto que as fibras são as principais responsáveis pela resistência mecânica deste LMF.

A relativamente elevada razão $J_{m d} / J_{i d}$ do TiGra, variando no intervalo de 2 a 3 para as duas taxas de carregamento averiguadas e por todo e extenso intervalo de temperaturas amostrado nos ensaios de impacto (Figura 5), sinaliza que o potencial de seleção deste laminado estrutural para a indústria da construção aeronáutica se justifica mais pela sua resistência à propagação do que à iniciação da fratura dinâmica.

Há de se destacar que o laminado híbrido TiGra possui um percentual de fibras de reforço na direção principal $\left(0^{\circ}\right)$ cerca de $20 \%$ inferior ao dos compósitos C-EPX ${ }^{[4]}$. Pode-se, portanto, postular que o desempenho do TiGra perante os seus concorrentes poderia ser significativamente melhorado caso se empregassem, na sua confecção, a mesma fração volumétrica de fibras naquela direção que a utilizada nos laminados C-EPX.

\section{Efeito da temperatura de ensaio}

Para uma velocidade de impacto de 2,25 m/s, a Figura 3 mostra que um aumento na temperatura de ensaio causa um significativo decréscimo em ambas as tenacidades $J_{i d}$ e $J_{m d}$ do laminado TiGra, indicando um enfraquecimento do mecanismo de tenacificação deste material durante seu aquecimento. Em estudo anterior dos autores $^{[4]}$, houve fortes indícios de que, para o laminado compósito C-EPX TP, o mecanismo de tenacificação afetado pela temperatura é o de delaminação, e a razão seria o surgimento de tensões residuais entre lâminas ou camadas justapostas do laminado, cujas fibras estão dispostas ortogonalmente entre si. Segundo o raciocínio desenvolvido, estas tensões interlaminares resultariam basicamente de variações dimensionais diferenciais induzidas termicamente, podendo favorecer a delaminação caso provocassem tração na direção da espessura do laminado. Arguiu-se, na ocasião, que este favorecimento da delaminação, causando em ultima instância à tenacificação do material, seria suprimido na medida em que a temperatura é incrementada, reduzindo assim ambos os valores de $J_{i d}$ e $J_{m d}$.

$\mathrm{O}$ mesmo fenômeno acima descrito pode estar ocorrendo também no TiGra. Como neste laminado o reforço fibroso de grafite está orientado em uma única direção $\left(0^{\circ}\right)$, elevados gradientes de variação dimensional termicamente induzida provavelmente

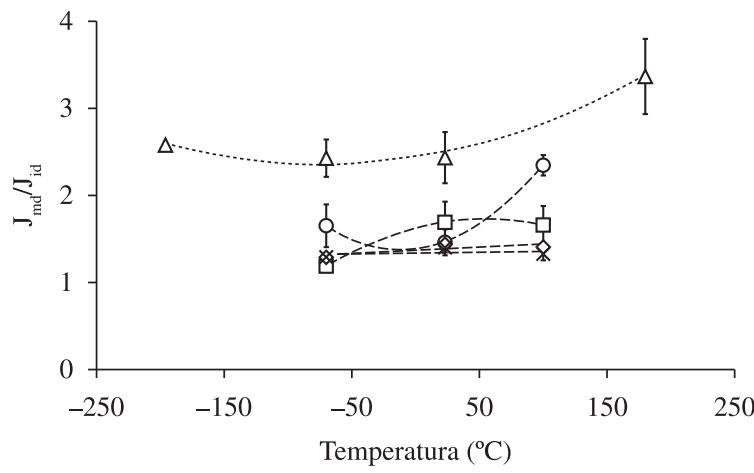

$\Delta$ TiGra $2,25 \mathrm{~m} / \mathrm{s} \circ \mathrm{FT} 120 \square \mathrm{FT} 180 \diamond \mathrm{TC} 120 \times \mathrm{TC} 180$

(a)

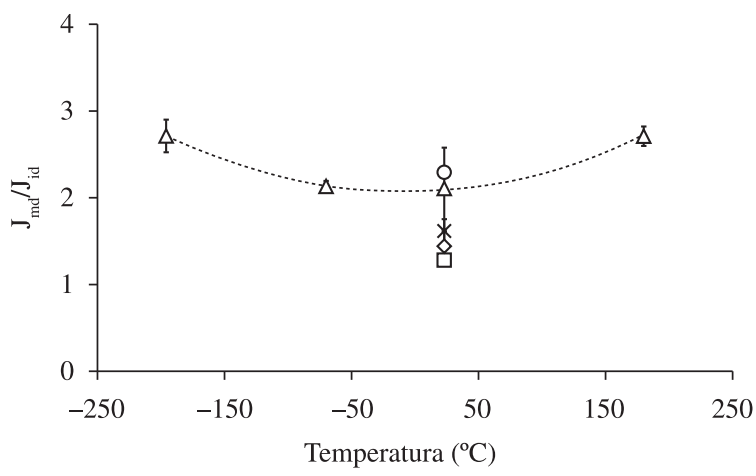

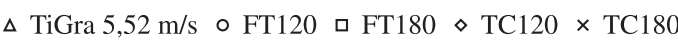

(b)

Figura 5. Razão $J_{m d} / J_{i d}$ calculada para os laminados avaliados em diversas temperaturas sob velocidade de impacto de: a) $2,25 \mathrm{~m} / \mathrm{s}$; e b) $5,52 \mathrm{~m} / \mathrm{s}$.

surjam nas interfaces metal/compósito, já que o coeficiente de expansão/contração térmica do Ti é muito superior ao das fibras de grafite, respectivamente $+8,8$ contra $-0,8 \mu \mathrm{m} .{ }^{\circ} \mathrm{C}^{-1}$, incentivando a ocorrência de delaminações nas mais baixas temperaturas de ensaio, $\mathrm{e}$, consequentemente, maiores valores de $J_{i d}$ e $J_{m d}$. Avaliações mais rigorosas deverão ser conduzidas para confirmar, ou não, tal hipótese. 
Nos impactos a $5,52 \mathrm{~m} / \mathrm{s}$, o laminado TiGra pode ser considerado praticamente insensível à variação da temperatura, donde pode-se inferir que taxas mais elevadas de carregamento não favoreçam o desenvolvimento do mecanismo tenacificador de delaminação, mas sim e somente a fratura translaminar em modo I de abertura do entalhe.

Resultados da análise estatística permitiram concluir que a tenacidade elasto-plástica $J_{m d}$ do laminado TiGra, o qual inclui certa parcela do processo de propagação de danos, é o critério de fratura mais provavelmente afetado pela variação da temperatura sob as duas taxas de carregamento.

Das análises, concluiu-se também que para o laminado TiGra, a razão entre tenacidades elasto-plásticas $J_{m d} / J_{i d}$ é mais influenciada pela temperatura de ensaio sob a taxa de carregamento de $5,52 \mathrm{~m} / \mathrm{s}$ do que a $2,25 \mathrm{~m} / \mathrm{s}$.

\section{Efeito da taxa de carregamento}

A Figura 3 indica um efeito benéfico do aumento da taxa de carregamento na tenacidade $J_{i d}$ do laminado TiGra. Este comportamento é tipicamente observado em materiais metálicos de natureza dúctil, cujo processo de fratura é controlado por deformação. Este fato é concordante com a massiva presença do titânio (55\% em massa), bem como do polímero PEEK (20\% em massa) no LMF.

Com relação ao critério de carga máxima $J_{m d}$, o TiGra apresenta claramente uma sensibilidade positiva ao incremento na taxa de carregamento em praticamente todo o intervalo avaliado de temperaturas.

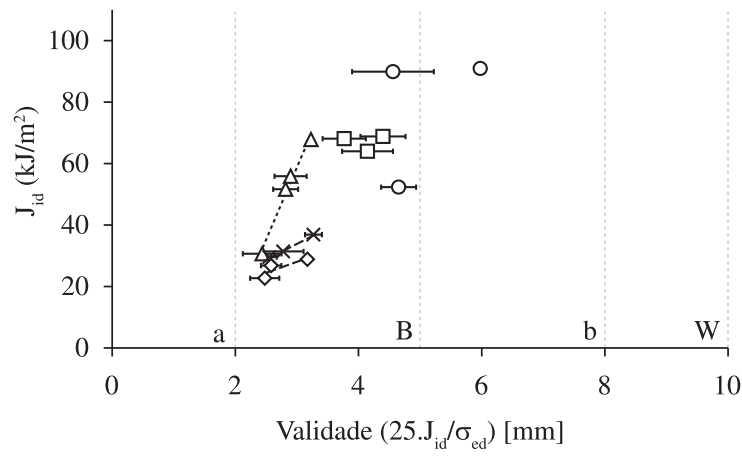

$\Delta$ TiGra 2,25 m/s o FT120 $\square$ FT180 $\diamond$ TC120 × TC180

(a)

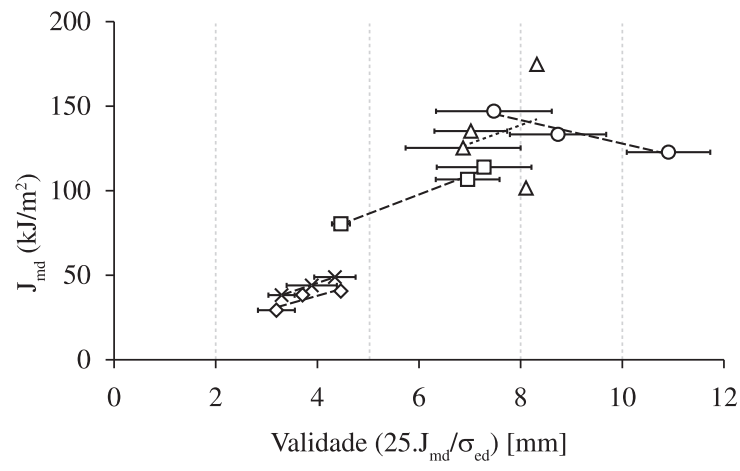

$\Delta$ TiGra $2,25 \mathrm{~m} / \mathrm{s}$ ○ FT120 $\square$ FT180 $\diamond \mathrm{TC} 120 \times \mathrm{TC} 180$
Interessante observar que, à semelhança do TiGra, o laminado C-EPX FT120 apresenta uma significativa tenacificação devida ao aumentonavelocidadedeimpacto.Estasimilaridadedecomportamentos de fratura entre os laminados TiGra e C-EPX FT120 já foi abordada no item anterior acerca do efeito da temperatura de ensaio, quando se inferiu a possibilidade de as delaminações controlarem o mecanismo de fratura de ambos os materiais. Analogamente, poder-se-ia agora postular que a tenacificação sob mais elevadas taxas de carregamento também seja fruto da facilitação da ocorrência de delaminação sob as condições mais severas de impacto.

A análise estatística permitiu inferir que a tenacidade elasto-plástica $J_{i d}$ do laminado TiGra é o critério de fratura mais provavelmente afetado pela variação da velocidade de impacto nas várias temperatura de ensaio empregadas.

\section{Validação dos resultados de tenacidade}

A Figura 6 mostra os resultados gráficos do processo de verificação da validação dos valores de tenacidade $J_{i d}$ e $J_{m d}$ por intermédio da aplicação da Equação 2, que corresponde matematicamente a uma inequação, visto definir uma desigualdade. $\mathrm{O}$ eixo das abscissas representa os valores numéricos obtidos por intermédio do termo situado do lado direito da Equação 2. Portanto, o procedimento consiste na comparação destes valores calculados com base nos resultados experimentais de, respectivamente, $J_{i d}$ e $J_{m d}$ com as dimensões significativas dos cdps ensaiados sob impacto, a saber: espessura $(B=5 \mathrm{~mm})$, ligamento $(b=8 \mathrm{~mm})$, largura ( $W=10 \mathrm{~mm})$, e comprimento do entalhe $(a=2 \mathrm{~mm})$, que são grafadas na Figura 6 a.

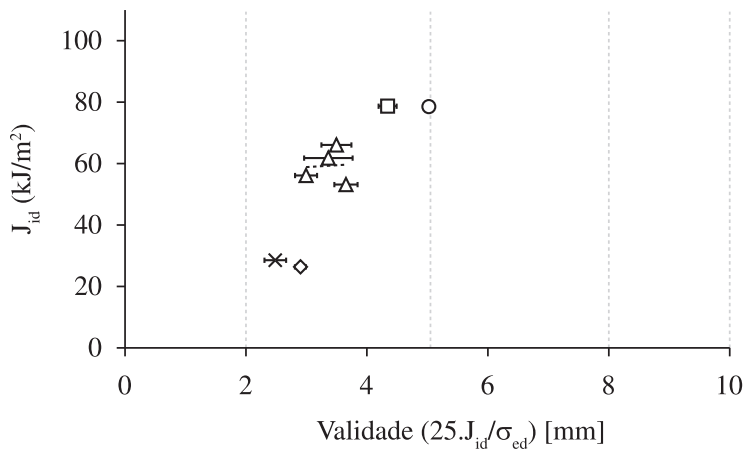

$\Delta$ TiGra $5,52 \mathrm{~m} / \mathrm{s}$ ○ FT120 $\square$ FT180 $\diamond \mathrm{TC} 120 \times \mathrm{TC} 180$

(b)

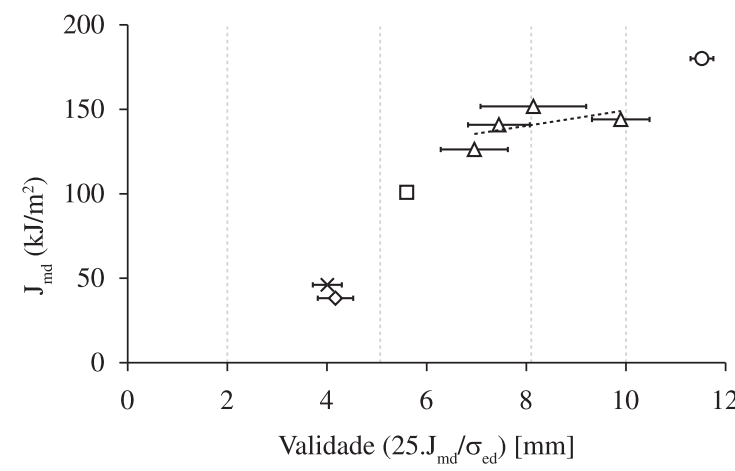

$\Delta$ TiGra $5,52 \mathrm{~m} / \mathrm{s} \circ \mathrm{FT} 120 \square \mathrm{FT} 180 \diamond \mathrm{TC} 120 \times \mathrm{TC} 180$

(c)

(d)

Figura 6. Critérios de validade das tenacidades $J_{i d}$ e $J_{m d}$ : a) $J_{i d}$ a $2,25 \mathrm{~m} / \mathrm{s} ;$ b) $J_{i d}$ a $5,52 \mathrm{~m} / \mathrm{s} ;$ c) $J_{m d}$ a 2,25 m/s; e d) $J_{m d}$ a $5,52 \mathrm{~m} / \mathrm{s}$. Note o comportamento tipo lei de potência das nuvens de pontos de dados. 
Desta forma, caso os pontos de dados assim calculados se encontrem à esquerda do respectivo valor do critério de validação aplicado à Equação 2, seja ele $B, b, W$ ou $a$, os pontos de dados $J_{i d}$ e $J_{m d}$ estão automaticamente validados segundo aquele critério em particular, visto que a condição imposta pela Equação 2 é plenamente satisfeita. Caso contrário, ou seja, se os pontos de dados estão localizados à direita do critério de validação empregado, o requisito de dimensão mínima do corpo de prova não é satisfeito para estabelecer $J_{i d}$ e $J_{m d}$ como verdadeiras propriedades do material naquela condição de ensaio, já que uma condição minimamente admissível de predominância de deformação-plana à frente do defeito não pode ser garantida.

Os valores médios das validades J obtidas a partir de três ensaios para cada condição experimental são plotados, com os respectivos desvios-padrão fornecidos na forma de barras horizontais.

Resultados previamente obtidos para quatro laminados C-EPX são plotados como linha de base para os valores determinados para o TiGra.

Observa-se na Figura 6a, b que, à exceção do comprimento do entalhe $(a=2 \mathrm{~mm})$, o laminado TiGra satisfaz plenamente os critérios estabelecidos em termos de dimensões mínimas necessárias do cdp para o desenvolvimento de uma condição de deformação predominantemente plana à frente do entalhe. Desta forma, conclui-se que a espessura $(B)$, o ligamento $(b)$ e a largura do cdp $(W)$ são suficientemente robustos para garantir $J_{i d}$ como uma verdadeira propriedade do material. Nestas circunstâncias, $J_{i d}$ passa a assumir a denominação $J_{I d}$.

Como esperado, os valores $J_{m d}$, por já embutirem em si uma boa parcela do estágio de propagação da fratura (que, a rigor, é iniciada em $J_{i d}$ ) e, consequentemente, estarem associados a uma zona de processos fratura mais ampla e difusa se comparada àquela vinculada ao critério $J_{i d}$, tendem a violar, com maior frequência e extensão, os critérios de validade impostos em termos de dimensões dos cdps Charpy ensaiados. Entretanto, mesmo para $J_{m d}$, é garantida para o TiGra uma condição de deformação predominantemente plana à frente do entalhe, de sorte que este critério pode ser considerado uma propriedade intrínseca do material.

Em termos estatísticos, o processo de validação das tenacidades elasto-plásticas $J_{i d}$ e $J_{m d}$ do laminado TiGra é afetado pela variação da temperatura de ensaio sob a taxa de carregamento de $2,25 \mathrm{~m} / \mathrm{s}$; porém esta dependência tende a decrescer na medida que a velocidade de impacto é incrementada para $5,52 \mathrm{~m} / \mathrm{s}$.

Verificou-se, também estatisticamente, que o processo de validação da tenacidade elasto-plástica $J_{i d}$ do laminado TiGra é muito provavelmente afetado pela variação da velocidade de impacto nas várias temperatura de ensaio empregadas no estudo; por outro lado, o processo de validação da tenacidade elasto-plástica $J_{m d}$ do laminado híbrido praticamente não depende da velocidade de carregamento mecânico em quaisquer das temperaturas amostradas na pesquisa.

\section{Conclusões}

A tenacidade integral-J à fratura elasto-plástica dinâmica do laminado híbrido metal-fibra Titânio-Grafite (TiGra) foi determinada a partir de ensaios de impacto Charpy instrumentado e comparada à de laminados convencionais carbono-epóxi (C-EPX). As principais conclusões do presente estudo são:

- O laminado TiGra exibe valores de tenacidade real à fratura $J_{i d}$ muito inferiores aos dos laminados C-EPX fita, porém significativamente superiores aos dos laminados C-EPX tecido;

- Os elevados valores de tenacidade de carga máxima $J_{m d}$ do TiGra, relativamente à tenacidade real à fratura $J_{i d}$ do material, indicam que o emprego do laminado-híbrido metal-fibra se justifica mais pela sua resistência à propagação do que à iniciação da fratura dinâmica;
- Observou-se que as tenacidades $J_{i d}$ e $J_{m d}$ à fratura translaminar do laminado TiGra são controladas pela intercedência de delaminacões, as quais podem se desenvolver já no início do processo de fratura em modo de abertura I do entalhe;

- Salvo o critério de validação de J com base no comprimento do entalhe, o laminado TiGra satisfez plenamente os requisitos quanto ao tamanho mínimo do corpo de prova para garantir ambos $J_{i d}$ e $J_{m d}$ como verdadeiras propriedades do material; e

- A metodologia desenvolvida pode ser de grande utilidade na etapa de seleção de materiais de engenharia para os quais as tenacidades à iniciação e à propagação de fratura sob cargas de impacto de baixa velocidade, porém com elevada inércia, sejam critérios do projeto estrutural aeronáutico.

\section{Agradecimentos}

Às agências de fomento FIPAI e CAPES, e à empresa MIB (Materials Institute of Brazil) pelo suporte financeiro, ao Prof. J.W. Cantwell da Universidade de Liverpool (UK) por disponibilizar laboratórios e equipamentos para a confecção do LMF-TiGra, ao Mestre em Ciência e Engenharia de Materiais D.S. Zanetti por compartilhar seus resultados experimentais, e ao hoje Engenheiro Aeronáutico G.A. Teti por sua assistência nos ensaios de impacto. Os professores D. Spinelli e W.W. Bose do NEMAF-EESC-USP são também reconhecidos por permitirem o uso do sistema instrumentado de ensaios de impacto Charpy.

\section{Referências Bibliográficas}

1. Burianek, D. A. \& Spearing, S. M. - Comp. Sci. Technol., 62, p.607 (2002).

2. Abrate, S. - Appl. Mech. Rev., 44, p.155 (1991).

3. Rice, J. R. - ASTM STP, 536, p.231 (1973).

4. Tarpani J. R. \& Gatti, M. C. A. - Polímeros (in press).

5. Alves, M.; Chaves, C. \& Birch, R.S. - "Impact on aircraft" in: Anais do $17^{\text {th }}$ Brazilian Congress on Mechanical Engineering, São Paulo- SP (2003).

6. Tarpani, J. R. - "Tenacidade ao impacto Charpy de materiais de construção aeronáutica”, Tese de Livre-Docência, Universidade de São Paulo, Escola de Engenharia de São Carlos, Brasil (2007).

7. Yu, A. \& Gupta, V. - Comp. Sci. Technol., 58, p.154 (1998).

8. Halli, R. B. \& Hager, J. W. J. - Compos. Mater., 30, p.1922 (1996).

9. Gatti, M. C. A.; Silva, R. V. \& Tarpani J. R. - Rev. Mat., 11, p.332 (2006).

10. Swain, R. E.; Bakis, C. E. \& Reifsnider, K. L. - ASTM STP, 1156, p.552 (1993).

11. Underwood, J. H. \& Kortschot, M. T. - “AD-A280 101 Technical Report ARCCBTR-94010”, US Army Armament Research, Development and Engineering Center, Watervliet, N.Y., (1994).

12. Masters, J. E. - “NASA Contractor Report 201728”, (1997).

13. Castrodeza, E. M.; Ipina, J. E. P. \& Bastian, F. L. - Fatigue Fract. Eng. Mater. Struct., 25, p.999 (2002).

14. Fernandez-Cantelli, A.; Arguelles, A.; Vina, J.; Ramulu, M. \& Kobayashi, A. S. Comp. Sci. Technol., 62, p.1315 (2002).

15. American Society for Testing and Materials. - "Standard test method for linearelastic plain-strain fracture toughness $K_{I c}$ of metallic materials, Designation ASTM-E399", 2005.

16. Kobayahi, T.; Yamamoto, I. \& Niinomi, M. J. - Test. Eval., 21, p.145 (1993).

17. Zhang, X. P. \& Shi, Y. W. - Int. J. Fract., 81, p.195 (1996).

18. Tronskar, J. P. \& Lai, M. - Eng. Fract. Mech., 69, p.321 (2002).

19. American Society for Testing and Materials. - "Standard test method for measurement of fracture toughness, Designation ASTM-E1820", 2005.

20. Server, W. L. J. - Test. Eval., 6, p.29 (1978).

21. Costa Neto, P. L. O. - "Estatística”, Edgar Blucher, São Paulo (1977).

22. Spiegel, M. R. - "Estatística”, Mc Graw Hill, São Paulo (1970).

23. Achcar, J. A. \& Rodrigues, J. - "Introdução à estatística para ciência e tecnologia”, EdUSP, São Paulo (2000).

Enviado: $22 / 02 / 10$

Aceito: $13 / 04 / 10$

DOI: $10.1590 / \mathrm{S} 0104-14282010005000041$ 